\title{
Prediction of ciclesonide binding site on middle-east respiratory syndrome coronavirus nsp15 multimer by molecular dynamics simulations
}

\author{
Shun Sakuraba and Hidetoshi Kono*
}

\begin{abstract}
Ciclesonide, a corticosteroid drug, was recently reported to inhibit the replication of Middle East respiratory syndrome coronavirus (MERS-CoV) as well as severe acute respiratory syndrome coronavirus 2 (SARS-CoV-2). To elucidate the molecular mechanism of the growth inhibition, we performed rigorous simulations to predict the pose of ciclesonide bound to MERSCoV's non-structural protein 15 (nsp15). Using the current state-of-the-art molecular simulation technique, we predicted the binding poses of ciclesonide and its metabolite to MERS-CoV's nsp15 pentamer. Effects of mutations of SARS CoV-2 nsp15 on the bindings are also discussed.
\end{abstract}

\section{Introduction}

The ongoing outbreak of COVID-19 was declared as a public health emergency of international concern by the World Health Organization. At the moment of writing this paper (25th December 2020), 77 million confirmed cases were reported worldwide. Effective antiviral agents to its causative virus, severe acute respiratory syndrome coronavirus 2 (SARS-CoV-2), are highly anticipated. Matsuyama et al. [1, 2] reported that ciclesonide (Fig. 1), which is a corticosteroid, blocks the viral particle replication of both SARS-CoV-2 and Middle-East Respiratory Syndrome (MERS)-CoV. In their evaluation with MERS-CoV mutants, it has been shown that a point mutation Ala25 $\rightarrow$ Val in MERS-CoV's non-structural protein 15 (nsp15) develops resistance to ciclesonide. It has been

*Institute for Quantum Life Science, National Institutes for Quantum and Radiological Research and Technology, Umemidai 8-1-7, Kidugawa, Kyoto 619-0215, Japan; \{sakuraba.shun, kono.hidetoshi\}@qst.go.jp. 
known that nsp15s of SARS-CoV's, SARS-CoV2's, and MERS-CoV's form homo-hexamers.[3, 4, 5] Furthermore, in MERS-CoV nsp15, the residue 25 is placed at the interface of the nsp15 hexamer. Thus, it has been suggested that ciclesonide interacts with nsp15 during viral biogenesis.[1,2] However, their precise molecular mechanism has been yet to be uncovered.

In the present study, we investigated the interaction between nsp15 multimer and ciclesonide by in-silico docking and molecular dynamics (MD) simulations with free-energy calculations. From the docking analysis, we predicted several putative binding sites. These binding sites were screened using rigorous calculations with the extended ensemble molecular dynamics simulations. We further performed the free-energy perturbation calculation that can evaluate the absolute binding free energy for identifying the correct binding site. The binding free energy between ciclesonide and the MERSCoV nsp15 was strong enough to achieve the reported inhibition level.

\section{Methods}

\subsection{Molecular Docking}

Molecular docking was first performed to determine the putative binding sites. Two ligand molecules were considered: (1) ciclesonide (Fig.1(a)), and (2) its digestive product, deesterified ciclesonide (des-ciclesonide; Fig. 1(b)). The initial structure of MERS-CoV's was taken from Zhang et al. [4] (Protein Data Bank ID: 5YVD). As we explained in Zhang et al. [4], nsp15 of MERS-CoV is considered to form a homohexamer. We prepared two structures: (a) a trimer of nsp15 proteins and (b) a pentamer of nsp15 proteins. The trimer and the hexamer structures were first constructed by applying symmetric operations to original structures to form a proper biological assembly. The pentamer structure was then constructed by removing one of the nsp $15 \mathrm{~s}$ from a hexameric structure. For both protein structures, we specified regions centered at Ala25, which was a reported mutation in a ciclesonide-resistant mutant. The docking calculation was performed with AutoDock Vina[6] with an "exhaustiveness" parameter of 2000 and the box size of $40 \AA \times 40 \AA \times 40 \AA$. Because we docked the ligand to the pentamer, we used three different bounding boxes centering Ala25 of three different chains near the missing monomer; we merged the results according to the docking score during a post-processing phase. For each combination of the protein target and ligand, the top nine poses were selected; in the trimer's case, three poses remained after removing the duplicates due to the symmetry. These top poses were used in the subsequent analysis.

We also performed the docking between SARS-CoV-2 nsp15 monomer [5] and des-ciclesonide as 
presented in Ref. [7]. However, we could not reproduce the reported docking score nor the docking pose presented in the literature. Combined with the contradiction to mutant analysis, we did not use any monomer-based structures in the subsequent analysis.

\subsection{Molecular Dynamics Simulations}

Molecular dynamics simulations were performed to relax the initial poses obtained. It is well known that structures sampled in the molecular simulations tend to be trapped at the initial configurations. To find appropriate docked structures, we employed a generalized replica exchange with solute tempering (gREST) molecular dynamics[8, 9] simulations. This method enables us to sample configurations from the canonical ensemble within a reasonable amount of computational resources. The protein was modeled using AMBERff14SB force field.[10, 11] Histidines at residues $66,246,259$, and 273 were protonated at $\mathrm{N} \delta$ sites, while other histidines were protonated at $\mathrm{N} \epsilon$ sites. The force fields for ciclesonide and des-ciclesonide were constructed with General Amber Force Field [12] version 2 (GAFF2). The charges of atoms were determined using the restrained electrostatic potential (RESP) $[13,14]$ method with HF $/ 6-31 \mathrm{G}^{*}$ level of quantum calculation. The protein multimer and the ligand were solvated with $150 \mathrm{mM} \mathrm{NaCl}$ solution. The rhombic dodecahedron periodic boundary condition was used. The periodic boundaries were at least $10 \AA$ away from proteins. The force field was then converted into GROMACS molecular dynamics software format via acpype,[15] and the simulations were performed by GROMACS 2019.[16] We used our in-house topology generator to employ the gREST simulation on GROMACS (https://bitbucket.org/shun.sakuraba/rest2py/src/master/). Each system was equilibrated by a $100 \mathrm{ps}$ run of constant-volume and constant-temperature (NVT) simulation, then another $100 \mathrm{ps}$ run of constant-pressure and constant-temperature (NPT) simulation. After the NPT equilibration runs, gREST simulations were performed with 8 replicas, with "hot" region being set to the ligand and the residues within $8 \AA$ from the ligand in the initial docking pose. Only the dihedral angle parameters were scaled in the gREST simulation from 100\% (replica 0, $300 \mathrm{~K}$ ) to $50 \%$ (replica 7, 600 K equivalent). gREST simulations were performed for $4 \mathrm{~ns}$ (32 ns in total for each pose). Obtained poses are presented in the supplemental material. Figures 2-4 were generated using VMD.[17]

The interaction between ligands and the protein was investigated. Snapshots from the last $2 \mathrm{~ns}$ (replica 0) of the gREST runs were further analyzed from two aspects, (1) van der Waals contacts between the ligand and the protein, and (2) hydrogen bonds between the ligand and the protein. For the van der Waals contacts, we counted the number of snapshots such that the target amino acid 
residue is within $4 \AA$ of any ligand atoms. We listed the residues which had interactions in more than $80 \%$ of the snapshots. For the hydrogen bonds, we defined that donor-hydrogen-acceptor atoms are making hydrogen bonds if the distance between the hydrogen and the acceptor is less than $2.5 \AA$, and the angle between donor-hydrogen-acceptor atoms is greater than $120^{\circ}$. To combine hydrogen bonds involving chemically equivalent atoms (e.g., OG1 and OG2 of Asp), we aggregated the hydrogen bonds binder in an amino acid into two groups, namely mainchain and sidechain. We also searched hydrogen bonds such that water molecules are bridging the protein and the ligand.

\subsection{Free Energy Perturbation Calculation}

After the putative relaxed conformations of the MERS nsp15-(des-)ciclesonide complex were obtained, we quantitatively confirmed the binding stability by the free-energy perturbation calculations. It has been demonstrated that using absolute binding free energy calculation after obtaining the docked structure can distinguish possible binding modes among docked structures. $[9,18]$ We used the double annihilation procedure to compute the binding free energy. Absolute binding free energies were estimated using the virtual bond algorithm.[19] We followed a protocol similar to Li et al. [20] combined with the Warp-Drive method[21] and the Hamiltonian replica exchange.[22, 23] The detailed procedure is presented in Appendix A. Final free energy values were calculated from the Bennett Acceptance Ratio method.[24] The dissociation constant $K_{d}$ was calculated using the relation

$$
K_{d}=C^{\circ} \exp \frac{\Delta G_{\text {bind }}^{\circ}}{R T}
$$

where $C^{\circ}$ is the standard concentration, which is in this research defined as $C^{\circ}=1 \mathrm{~mol} / \mathrm{L} .95 \%$ confidence interval was estimated from the variance of $\Delta G_{\text {bind }}^{\circ}$, assuming $\Delta G_{\text {bind }}^{\circ}$ obeys the normal distribution.

\subsection{Simulation of A25V mutants}

From the absolute binding free energy calculation, we identified a pose (see Subsection 3.1) for each ligand type. To elucidate the molecular mechanism of gaining the resistance for the nsp15 with the Ala25Val mutation, we performed the same absolute binding free-energy calculation with the Ala25Val mutation introduced at all the subunits of the pentamer. 


\subsection{Modeling SARS-CoV-2-ligand binding poses}

Ciclesonide inhibits SARS-CoV-2 replication as well as MERS-CoV.[1, 2] Due to the sequence similarity between MERS-CoV's nsp15 and SARS-CoV-2's nsp15, ciclesonide may also bind to the SARS-CoV-2 nsp15. We modeled the structure of a SARS-CoV-2 nsp15 pentamer[5] (Protein Data Bank ID: 6VWW) by superimposing nsp15 structures. Following the previous cases, the system was set up by the equilibration followed by a gREST relaxation and absolute binding free energy calculation.

\section{Results}

\subsection{Binding poses and its binding free energies}

We present the predicted binding free energies and the dissociation constants in Table 1 . In the case of pentamer-ciclesonide binding, pose 9 showed the best binding free energy, whereas for the pentamer-des-ciclesonide binding pose 19 showed the strongest binding. In both cases, the dissociation constants were as low as sub-micromolar levels, implying that the strong binding was achieved. For the case of the trimer, both ciclesonide and des-ciclesonide bound only weakly to the surface.

When the A25V mutations were introduced, the resulting binding free energy increased from -9.6 to $-8.7 \mathrm{kcal} / \mathrm{mol}$ for the case of ciclesonide and -10.4 to $-7.0 \mathrm{kcal} / \mathrm{mol}$ for the case of des-ciclesonide, destabilizing the bindings by $0.9 \mathrm{kcal} / \mathrm{mol}$ and $3.4 \mathrm{kcal} / \mathrm{mol}$, respectively (Table 1 ).

Finally, when the proteins were replaced with the SARS-CoV-2's nsp15, the binding became weak, and the binding free energy increased from -9.6 to $-8.8 \mathrm{kcal} / \mathrm{mol}$ for the case of ciclesonide and -10.4 to $-9.1 \mathrm{kcal} / \mathrm{mol}$ for the case of des-ciclesonide.

\subsection{Structural detail of the binding poses}

For the pentamer of MERS-CoV's nsp15, Fig. 2 shows the most stable poses of both ligands, i.e., ciclesonide pose 9 and des-ciclesonide pose 19 after the gREST relaxation. In both cases, the ligand resides at the interface of three monomers. The contact analysis and the hydrogen bonding investigation (Tables 2 and 3) shows that, for the case of ciclesonide pose 9 , ciclesonide bound mostly with hydrophobic interactions (only four hydrogen bonds), whereas des-ciclesonide pose 19 bound to the pentamer with two direct hydrogen bonds and five water-bridged hydrogen bonds.

In both models, an increase in the binding energy upon Ala25Val mutation can be structurally 
explained by the steric hindrance. The mutation of Ala25Val in chain C collided with Tyr104 of chain D, which supported des-ciclesonide backbone from the back, as shown in Figs. 3 and 4. Thus, the Ala25Val mutation is considered to hinder des-ciclesonide binding to the same pocket.

\subsection{Discussion}

It has been widely recognized that the absolute binding free energy calculation achieves the average error between the predicted binding free energies and the experimental free energies of $\sim 1 \mathrm{kcal} / \mathrm{mol}$. Since $1 \mathrm{kcal} / \mathrm{mol}$ difference corresponds to $\sim 5$ times difference in the binding constant, the predicted pentamer-(des-)ciclesonide binding may have less than $1 \mu \mathrm{M}$, which is considered strong enough to achieve the experimental inhibition constant of $20 \mu \mathrm{M}$ (though the concentration of the nsp15 in the cell is unknown). Increased (destabilized) binding free energy with Ala25Val also corroborates with the experimental fact that the Ala25Val to the nsp15 gains the resistance to ciclesonide. The predicted binding structure suggests that the sidechain of Lys34 contacts with ciclesonide, and ones of Thr48 and Tyr104 for des-ciclesonide, respectively. Thus the mutation to one of these residues may incite resistance to ciclesonide, though the mutation itself may also inhibit the multimerization even without (des-)ciclesonide.

Considering the increased number of hydrogen bonds and the increased stability of des-ciclesonide with pose 19 compared to ciclesonide, it is more likely that des-ciclesonide is the major active form of the inhibition. The hypothesis is also consistent with the time-of-addition assays in Matsuyama et al. [2, in Fig3]; when ciclesonide added to Vero cells at 5 hours after the postinfection and viral titer measured at 6 hours after the postinfection, the inhibition effect was almost indistinguishable from the control. Because the speed of deesterification of $5 \mu \mathrm{M}$ ciclesonide to des-ciclesonide is estimated to be $\sim 30 \%$ at 4 hours in the case of normal human bronchial epithelial cells,[25] the time-lag for the ciclesonide to be effective may represent the time required to convert ciclesonide into des-ciclesonide.

For the case of SARS-CoV-2,[2] the drug resistance has been reported to be obtained with mutations to nsp3 and nsp4. The result implies that the primary target of ciclesonide for SARS$\mathrm{CoV}-2$ is not nsp15. It is consistent with the increased (destabilized) binding free energy by replacing the MERS-CoV's nsp15 pentamer with the SARS-CoV-2's nsp15 pentamer.[2] However, with a relatively high stability (sub-micromolar level) of the binding between the SARS-CoV-2's nsp15 and (des-)ciclesonide, it is possible that a variant of (des-)ciclesonide effectively block SARS-CoV2's nsp15 multimerization. Thus, an inhibitor for SARS-CoV-2 may be designed by systematically 
testing variants of (des-)ciclesonide based on the current docking poses.

In the present simulation model, we only considered the trimer and the pentamer configuration for the protein. It may be possible that another monomer may bind to the pentamer with (des)ciclesonide and to form a non-symmetric, imperfect hexamer. In such a case, (des-)ciclesonide may have a lower binding free energy due to the tighter packing. Another possibility is that the ligand binds to a tetramer or non- $\mathrm{C}_{3}$-symmetric trimer, because in the current model, one ligand can bind at the interface of three monomers, and not all five monomers are required. There are possibilities to improve the prediction by considering more precise binding models, possibly with the help of experimental multimer state measurements.

Similar to the present research, a strategy to block the multimer binding may be universally applicable to inhibit enzymes of various pathogens. Upon the current pandemic of the SARS-CoV-2, drug repositioning targeting enzyme active centers has been performed. The present result suggests that in addition to the enzyme active center, the multimer interface may become a good target of inhibition. Indeed, a recent preprint article[26] has reported that Betulonic acid derivatives can inhibit nsp15 via the binding to multimer interface site. The development of a systematic screening procedure targeting these multimer interfaces may be beneficial to stand against different pathogens.

\section{Acknowledgment}

This research was supported by the Basis for Supporting Innovative Drug Discovery and Life Science (BINDS) project under Grant Number JP20am0101106j0004, Agency for Medical Research and Development (AMED), Japan to HK, and by the Grant-in-Aid for Scientific Research on Innovative Areas under Grant Number 19H05410, the Ministry of Education, Culture, Sports, Science and Technology (MEXT), Japan to SS. Simulations were performed on supercomputers at ACCMS, Kyoto University. Authors thank AMED BINDS in-silico project members for insightful comments. Authors declare no competing interests.

\section{References}

[1] Shutoku Matsuyama, Miyuki Kawase, Naganori Nao, Kazuya Shirato, Makoto Ujike, Wataru Kamitani, Masayuki Shimojima, and Shuetsu Fukushi. The inhaled corticosteroid ciclesonide 
blocks coronavirus RNA replication by targeting viral NSP15. bioRxiv, page 2020.03.11.987016, 2020. doi: 10.1101/2020.03.11.987016.

[2] Shutoku Matsuyama, Miyuki Kawase, Naganori Nao, Kazuya Shirato, Makoto Ujike, Wataru Kamitani, Masayuki Shimojima, and Shuetsu Fukushi. The Inhaled Steroid Ciclesonide Blocks SARS-CoV-2 RNA Replication by Targeting the Viral Replication-Transcription Complex in Cultured Cells. Journal of Virology, 95(1):e01648-20, 2020. doi: 10.1128/JVI.01648-20.

[3] Jeremiah S. Joseph, Kumar Singh Saikatendu, Vanitha Subramanian, Benjamin W. Neuman, Michael J. Buchmeier, Raymond C. Stevens, and Peter Kuhn. Crystal structure of a monomeric form of severe acute respiratory syndrome coronavirus endonuclease nsp15 suggests a role for hexamerization as an allosteric switch. Journal of Virology, 81(12):6700-6708, 2007. doi: 10. 1128/jvi.02817-06.

[4] Lianqi Zhang, Lei Li, Liming Yan, Zhenhua Ming, Zhihui Jia, Zhiyong Lou, and Zihe Rao. Structural and biochemical characterization of endoribonuclease nsp15 encoded by middle east respiratory syndrome coronavirus. Journal of Virology, 92(22):e00893-18, 2018. doi: 10.1128/ jvi.00893-18.

[5] Youngchang Kim, Robert Jedrzejczak, Natalia I. Maltseva, Mateusz Wilamowski, Michael Endres, Adam Godzik, Karolina Michalska, and Andrzej Joachimiak. Crystal structure of Nsp15 endoribonuclease NendoU from SARS-CoV-2. Protein Science, 29(7):1596-1605, 2020. doi: $10.1002 /$ pro. 3873 .

[6] Oleg Trott and Arthur J. Olson. AutoDock vina: Improving the speed and accuracy of docking with a new scoring function, efficient optimization, and multithreading. Journal of Computational Chemistry, 31(2):455-461, 2009. doi: 10.1002/jcc.21334.

[7] Hirokazu Kimura, Hiromu Kurusu, Mitsuru Sada, Daisuke Kurai, Koichi Murakami, Wataru Kamitani, Haruyoshi Tomita, Kazuhiko Katayama, and Akihide Ryo. Molecular pharmacology of ciclesonide against SARS-CoV-2. Journal of Allergy and Clinical Immunology, 146(2):330331, 2020. ISSN 0091-6749. doi: 10.1016/j.jaci.2020.05.029.

[8] Motoshi Kamiya and Yuji Sugita. Flexible selection of the solute region in replica exchange with solute tempering: Application to protein-folding simulations. The Journal of Chemical Physics, 149(7):072304, 2018. doi: 10.1063/1.5016222. 
[9] Hiraku Oshima, Suyong Re, and Yuji Sugita. Prediction of protein-ligand binding pose and affinity using the gREST+FEP method. Journal of Chemical Information and Modeling, 60 (11):5382-5394, 2020. doi: 10.1021/acs.jcim.0c00338.

[10] T. E. Cheatham III, P. Cieplak, and P. A. Kollman. A modified version of the cornell et al. force field with improved sugar pucker phases and helical repeat. J. Biomol. Struct. Dyn., 16: 845-862, 1999. doi: 10.1080/07391102.1999.10508297.

[11] James A. Maier, Carmenza Martinez, Koushik Kasavajhala, Lauren Wickstrom, Kevin E. Hauser, and Carlos Simmerling. ff14sb: Improving the accuracy of protein side chain and backbone parameters from ff99sb. Journal of Chemical Theory and Computation, 11(8):36963713, 2015. doi: 10.1021/acs.jctc.5b00255.

[12] Junmei Wang, Romain M. Wolf, James W. Caldwell, Peter A. Kollman, and David A. Case. Development and testing of a general amber force field. Journal of Computational Chemistry, 25(9):1157-1174, 2004. doi: 10.1002/jcc.20035.

[13] Christopher I. Bayly, Piotr Cieplak, Wendy Cornell, and Peter A. Kollman. A well-behaved electrostatic potential based method using charge restraints for deriving atomic charges: the RESP model. J. Phys. Chem., 97(40):10269-10280, 1993. doi: 10.1021/j100142a004.

[14] Piotr Cieplak, Wendy D Cornell, Christopher Bayly, and Peter A Kollman. Application of the multimolecule and multiconformational RESP methodology to biopolymers: Charge derivation for DNA, RNA, and proteins. J. Comput. Chem., 16(11):1357-1377, 1995.

[15] Alan W Sousa da Silva and Wim F Vranken. ACPYPE - AnteChamber PYthon parser interfacE. BMC Research Notes, 5(1):367, 2012. doi: 10.1186/1756-0500-5-367.

[16] Mark James Abraham, Teemu Murtola, Roland Schulz, Szilárd Páll, Jeremy C Smith, Berk Hess, and Erik Lindahl. Gromacs: High performance molecular simulations through multi-level parallelism from laptops to supercomputers. SoftwareX, 1:19-25, 2015.

[17] William Humphrey, Andrew Dalke, and Klaus Schulten. VMD - Visual Molecular Dynamics. Journal of Molecular Graphics, 14:33-38, 1996.

[18] Matteo Aldeghi, Alexander Heifetz, Michael J. Bodkin, Stefan Knapp, and Philip C. Biggin. Accurate calculation of the absolute free energy of binding for drug molecules. Chemical Science, 7(1):207-218, 2016. doi: 10.1039/c5sc02678d. 
[19] Stefan Boresch, Franz Tettinger, Martin Leitgeb, and Martin Karplus. Absolute binding free energies: a quantitative approach for their calculation. The Journal of Physical Chemistry B, 107(35):9535-9551, 2003. doi: 10.1021/jp0217839.

[20] Zhe Li, Xin Li, Yi-You Huang, Yaoxing Wu, Runduo Liu, Lingli Zhou, Yuxi Lin, Deyan Wu, Lei Zhang, Hao Liu, Ximing Xu, Kunqian Yu, Yuxia Zhang, Jun Cui, Chang-Guo Zhan, Xin Wang, and Hai-Bin Luo. Identify potent SARS-CoV-2 main protease inhibitors via accelerated free energy perturbation-based virtual screening of existing drugs. Proceedings of the National Academy of Sciences, 117(44):27381-27387, 2020. doi: 10.1073/pnas.2010470117.

[21] Toru Ekimoto, Tsutomu Yamane, and Mitsunori Ikeguchi. Elimination of finite-size effects on binding free energies via the warp-drive method. Journal of Chemical Theory and Computation, 14(12):6544-6559, 2018. doi: 10.1021/acs.jctc.8b00280.

[22] Hiroaki Fukunishi, Osamu Watanabe, and Shoji Takada. On the hamiltonian replica exchange method for efficient sampling of biomolecular systems: Application to protein structure prediction. J. Chem. Phys., 116(20):9058-9067, 2002. doi: 10.1063/1.1472510.

[23] Wei Jiang, Milan Hodoscek, and Benoît Roux. Computation of absolute hydration and binding free energy with free energy perturbation distributed replica-exchange molecular dynamics. Journal of Chemical Theory and Computation, 5(10):2583-2588, 2009. doi: 10.1021/ct900223z.

[24] Charles H Bennett. Efficient estimation of free energy differences from monte carlo data. Journal of Computational Physics, 22(2):245-268, 1976. doi: 10.1016/0021-9991(76)90078-4.

[25] Elaine Mutch, Ruediger Nave, Nigel McCracken, Karl Zech, and Faith M. Williams. The role of esterases in the metabolism of ciclesonide to desisobutyryl-ciclesonide in human tissue. Biochemical Pharmacology, 73(10):1657-1664, 2007. doi: 10.1016/j.bcp.2007.01.031.

[26] Besir Krasniqi, Annelies Stevaert, Benjamin Van Loy, Tien Nguyen, Joice Thomas, Julie Vandeput, Dirk Jochmans, Volker Thiel, Ronald Dijkman, Wim Dehaen, Arnout Voet, and Lieve Naesens. Betulonic acid derivatives inhibiting coronavirus replication in cell culture via the nsp15 endoribonuclease. bioRxiv, 2020. doi: 10.1101/2020.12.10.418996. 


\section{Appendices}

\section{A Detail of the free energy calculation protocol}

Figure 5 presents an overview of the free-energy calculation. As we explained in Section 2, the double annihilation method was used to calculate the free energy difference. In the double annihilation method, ligand charges and van der Waals interactions were scaled to 0 on both the ligand-solution system and the ligand-protein system. In the protein system, a restraining potential was added to fix the relative orientation of the ligand from the protein. The restraining potential was applied between three atoms in the protein and three atoms ("anchor atoms") in the ligand, with one bond restraint, two angle restraints, and three dihedral restraints. The force constant of the restraining potential was set to $1 \mathrm{kcal} / \mathrm{mol} / \AA^{2}, 1 \mathrm{kcal} / \mathrm{mol} / \mathrm{rad}^{2}$, and $1 \mathrm{kcal} / \mathrm{mol} / \mathrm{rad}^{2}$ for the bond restraint, the angle restraints, and the dihedral restraints, respectively. The target value of the restraint was calculated from the average of the distance, the angles, and the dihedrals in the gREST run. The free-energy difference due to the additional restraint is canceled using the analytical (approximate) correction $\Delta G_{\text {correction }}[19]$. Other $\Delta G$ s were calculated by free-energy perturbation with the Bennett acceptance ratio method.[24] To accelerate the conformational sampling, we used the Hamiltonian replica exchange method; for $\Delta G_{\text {complex,restr }}, 8$ replicas were used, and for other $\Delta G$ s 20 replicas were used. The discharging phases composed of $\Delta G_{\text {complex,charge }}$ and $\Delta G_{\text {ref,charge were calculated }}$ by using a single system containing both a protein-ligand complex and an other ligand molecule, and by discharging ligand bound to protein while charging the ligand in the solvent (Warp-Drive method [21]). In the annihilation phase of $\Delta G_{\text {complex,vdw }}$ and $\Delta G_{\text {ref,vdw }}$, van der Waals interactions were slowly removed (or added) using the soft-core potential (for the atoms with no van der Waals radius, the minimum soft-core van der Waals distance ("minimum $\sigma$ ") of 3.0 Åwas used; soft-core parameter (" $\alpha$ ") of 0.5 was used). Finally, restraint potentials were gradually removed to calculate $\Delta G_{\text {complex,restr }}$. For all calculations, 2 ns equilibration run was followed by 2 ns production run at 300 $\mathrm{K}, 1 \mathrm{~atm}$ condition. Langevin dynamics was used to control the temperature, and Parrinello-Rahman barostat was used. The cutoff distance was set to $10 \AA$ and smooth particle mesh Ewald method was used to compute the electrostatic interaction. The timestep was set to 2 fs and hydrogens were constrained to have constant bond lengths to heavy atoms. The total simulation time was $4 \mathrm{~ns} \times$ 68 replicas $\times 28$ poses $=7.6 \mu$ s. 


\section{List of Figures}

1 Structural formulae of (a) ciclesonide and (b) its active metabolite, des-ciclesonide. Numbers in parentheses represent the atom numbering used in Table 3. . . . . . . .

2 The lowest binding free energy poses (measured in free-energy perturbation calculation) of (a) ciclesonide-MERS CoV-nsp15 and (b) des-ciclesonide-MERS CoV-nsp15 complexes. Ligands are colored green. Monomers were labeled as: A (blue), B (red), $\mathrm{C}$ (pink), D (orange), and $\mathrm{E}$ (yellow). . . . . . . . . . . . . . .

3 Closeup view of the best binding pose for ciclesonide. (left) Residues making hydrogen bonds to ciclesonide were labeled. (right) Ala25 and Tyr104 positions represented by space-filled balls colored by purple and white, respectively. . . . . . . . . . . .

4 Closeup view of the best binding pose for des-ciclesonide. (left) Residues making hydrogen bonds to des-ciclesonide were labeled. (right) Ala25 and Tyr104 positions represented by space-filled balls colored by purple and white, respectively. . . . . .

5 A schematic diagram representing the free energy calculation protocol. The target quantity is the free energy difference represented as $\Delta G_{\text {bind }}^{\circ}$. The free-energy difference between the bottom two states $\Delta G_{\text {sys }}$ is exactly 0 due to the chemical state equivalence. The free-energy difference $\Delta G_{\text {correction }}$ is calculated by an analytical correction scheme. The difference of free-energy differences $\Delta G_{\text {complex,charge }}$ and $\Delta G_{\text {ligand,charge }}$ is calculated at once by the Warp-Drive method. Other $\Delta G$ values are calculated by the free-energy calculation with the Hamiltonian replica exchange method. . . . . . . . 
(a)

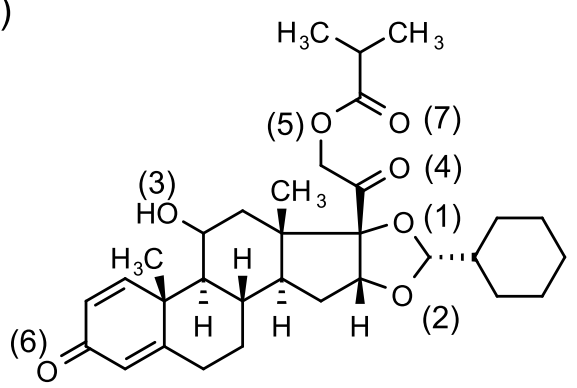

(b)

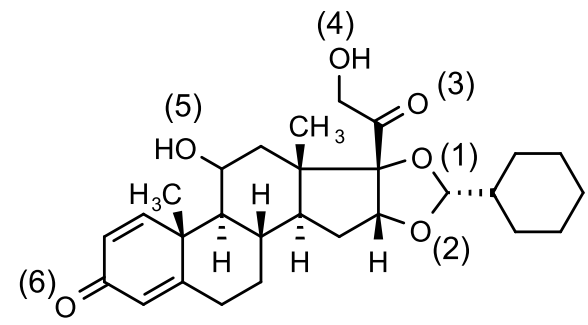

Figure 1: Structural formulae of (a) ciclesonide and (b) its active metabolite, des-ciclesonide. Numbers in parentheses represent the atom numbering used in Table 3. 
A
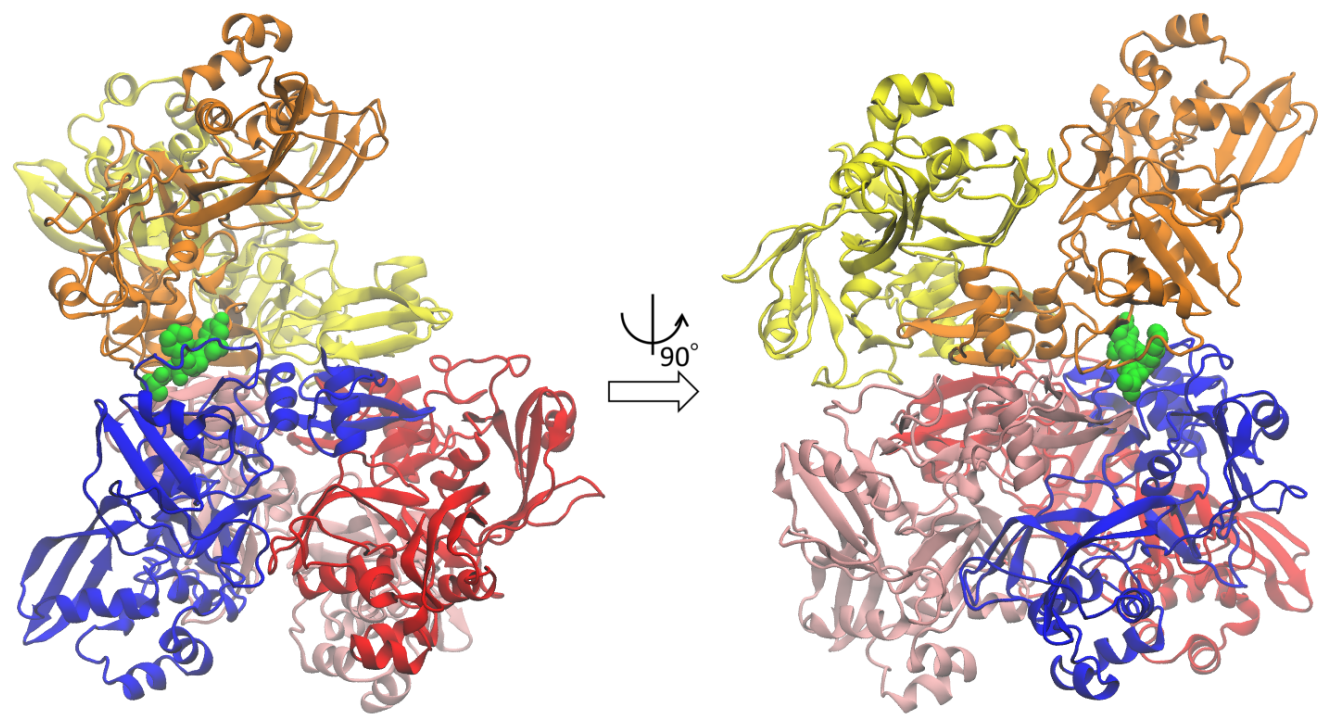

b
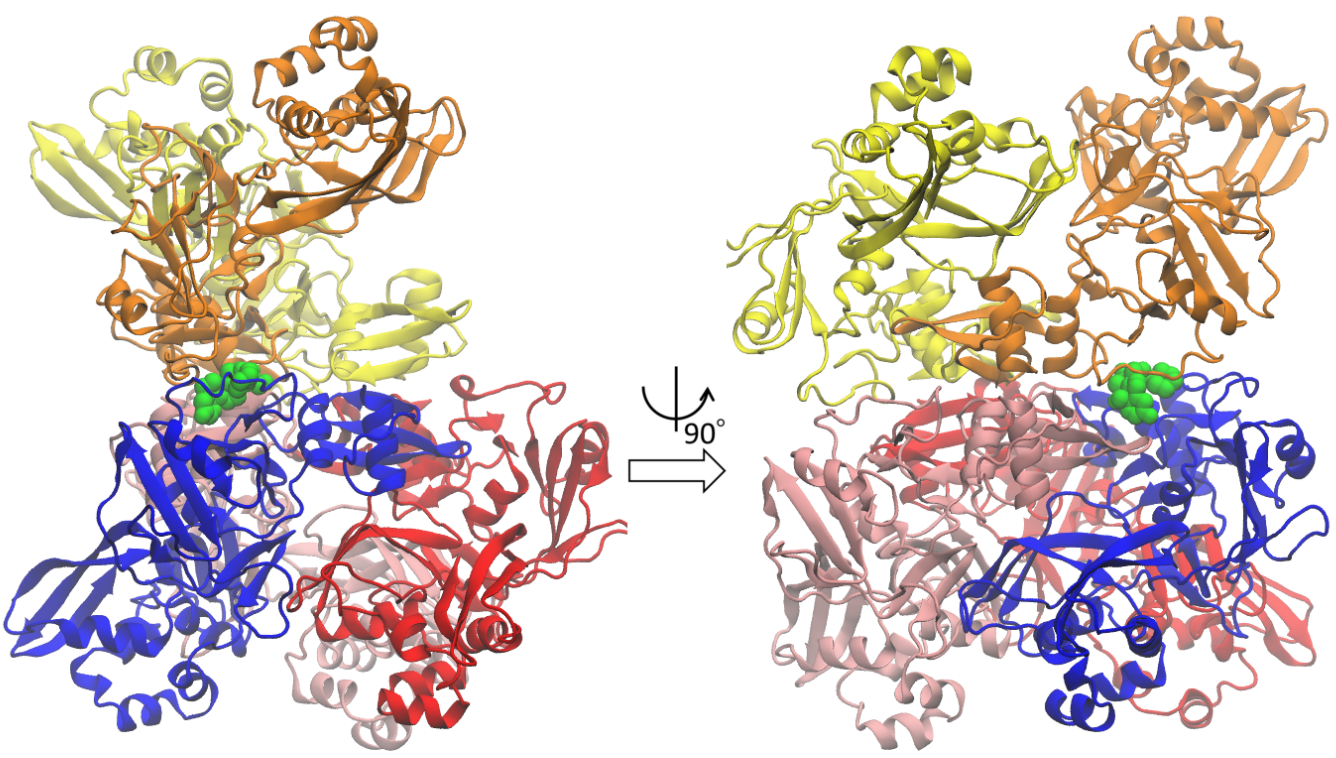

Figure 2: The lowest binding free energy poses (measured in free-energy perturbation calculation) of (a) ciclesonide-MERS CoV-nsp15 and (b) des-ciclesonide-MERS CoV-nsp15 complexes. Ligands are colored green. Monomers were labeled as: A (blue), B (red), C (pink), D (orange), and E (yellow). 


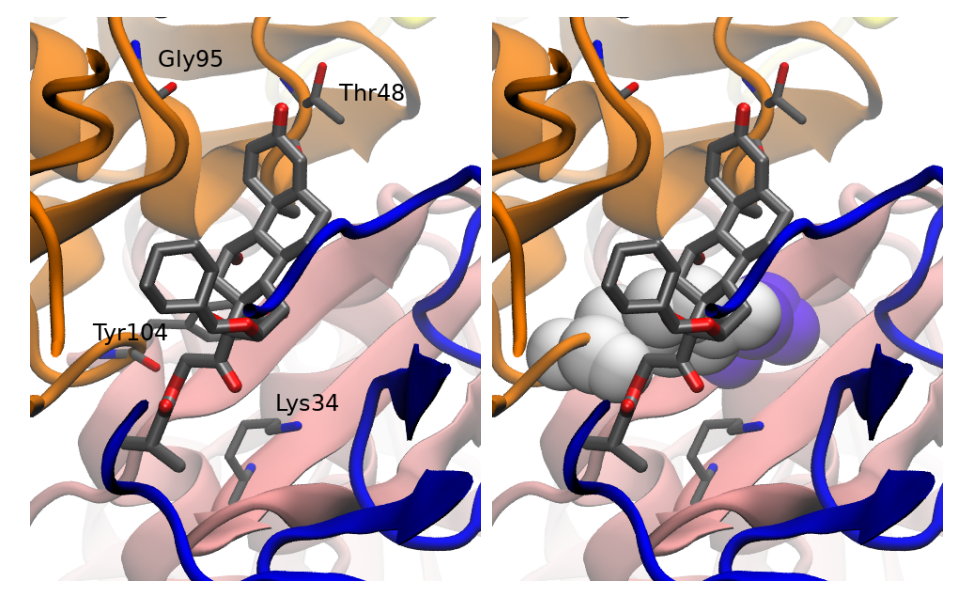

Figure 3: Closeup view of the best binding pose for ciclesonide. (left) Residues making hydrogen bonds to ciclesonide were labeled. (right) Ala25 and Tyr104 positions represented by space-filled balls colored by purple and white, respectively. 


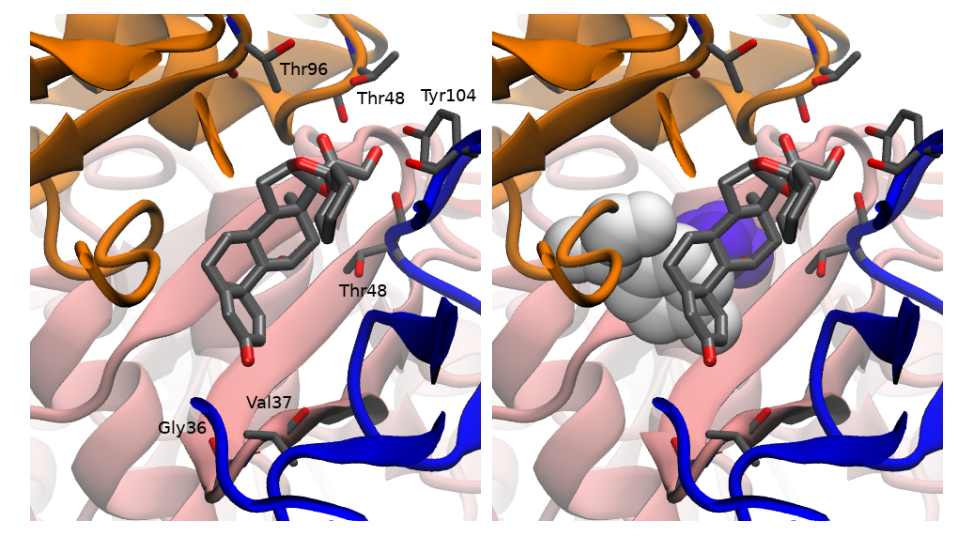

Figure 4: Closeup view of the best binding pose for des-ciclesonide. (left) Residues making hydrogen bonds to des-ciclesonide were labeled. (right) Ala25 and Tyr104 positions represented by space-filled balls colored by purple and white, respectively. 


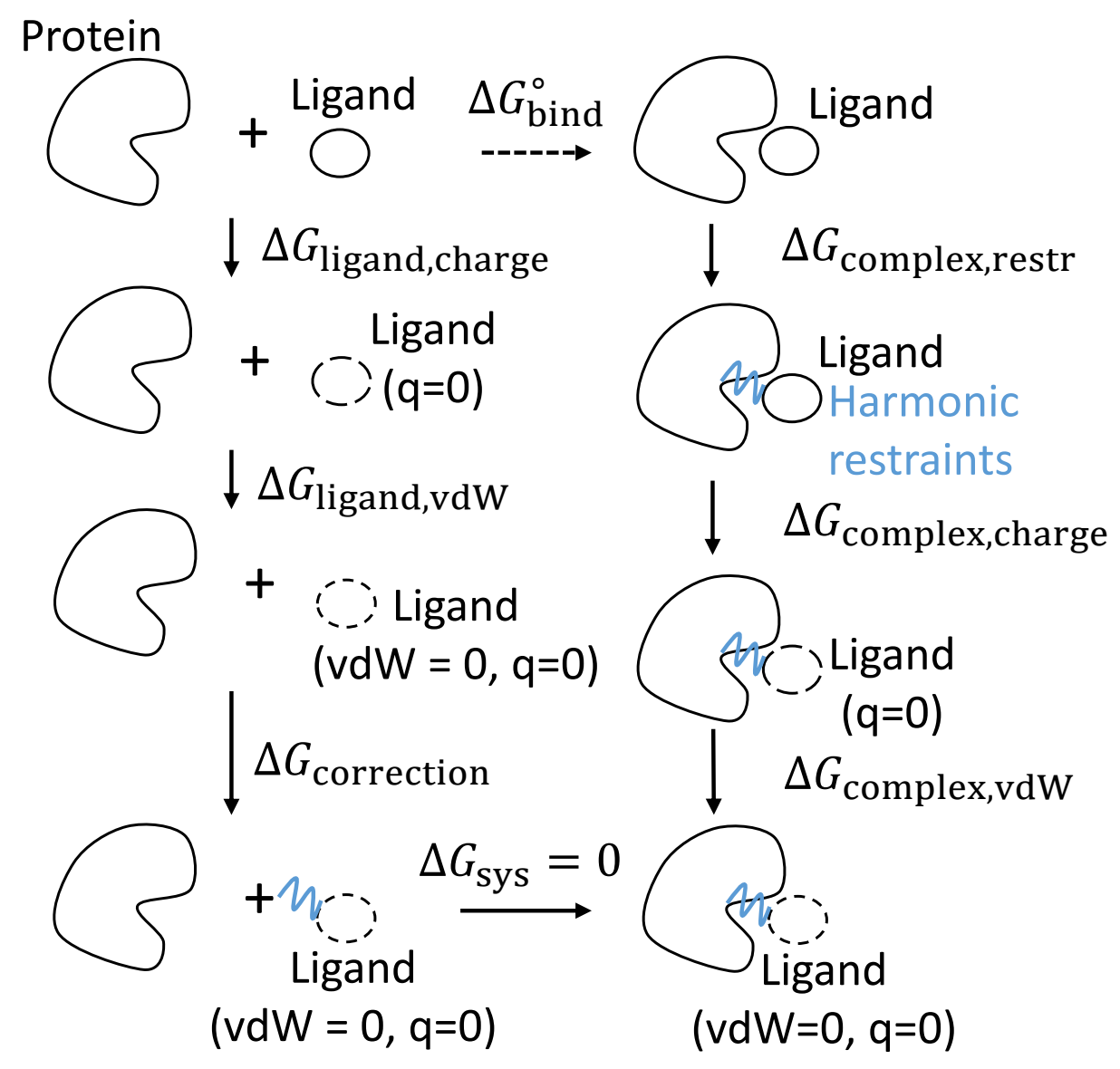

Figure 5: A schematic diagram representing the free energy calculation protocol. The target quantity is the free energy difference represented as $\Delta G_{\text {bind }}^{\circ}$. The free-energy difference between the bottom two states $\Delta G_{\text {sys }}$ is exactly 0 due to the chemical state equivalence. The free-energy difference $\Delta G_{\text {correction }}$ is calculated by an analytical correction scheme. The difference of free-energy differences $\Delta G_{\text {complex,charge }}$ and $\Delta G_{\text {ligand,charge }}$ is calculated at once by the Warp-Drive method. Other $\Delta G$ values are calculated by the free-energy calculation with the Hamiltonian replica exchange method. 


\section{List of Tables}

1 Estimated binding free-energy $\Delta G$ for each multimer protein and ligand combination. CIC: ciclesonide, DCI: des-ciclesonide. Confidence intervals (CIs) are presented as pairs of (lower, higher) intervals. Mutimer proteins are MERS-CoV's nsp15 except the last two rows. . . . . . . . . . . . . . . . . . . . .

2 Residues contacting with the ligand in more than $80 \%$ of the snapshots from gREST simulation. "\# Res." represents the residue number, and "Res. name" represents the residue name.

Hydrogen bonds between the protein and (des-)ciclesonide after the oREST relaxation. Hydrogen bonds that were observed in more than $10 \%$ of the snapshots were listed. Chain represents the protein monomer symbols as presented in Fig. 2. Ligand atom names of oxygen atoms corresponds to the number on oxygen atoms in Fig. 1. The row of "Water" being "yes" indicates that protein-ligand has water-bridged hydrogen bonds, i.e., one water atom simultaneously make hydrogen bonds to both ligand and protein in a single snapshot. . . . . . . . . . . . . . . . 


\begin{tabular}{|c|c|c|c|c|}
\hline Protein & Pose & Ligand & $\Delta G(\mathrm{kcal} / \mathrm{mol})$ & $K_{d}(\mu \mathrm{M}, 95 \% \mathrm{CI})$ \\
\hline pentamer & 1 & $\mathrm{CIC}$ & $-6.8 \pm 0.2$ & $10(4,20)$ \\
\hline pentamer & 2 & $\mathrm{CIC}$ & $-3.1 \pm 0.4$ & $5000(1700,18000)$ \\
\hline pentamer & 3 & $\mathrm{CIC}$ & $-3.1 \pm 0.4$ & $5000(1400,20000)$ \\
\hline pentamer & 4 & $\mathrm{CIC}$ & $-6.2 \pm 0.3$ & $30(11,70)$ \\
\hline pentamer & 5 & $\mathrm{CIC}$ & $-4.6 \pm 0.4$ & $400(100,1700)$ \\
\hline pentamer & 6 & $\mathrm{CIC}$ & $-2.1 \pm 0.4$ & $30000(8000,120000)$ \\
\hline pentamer & 7 & CIC & $-8.5 \pm 0.5$ & $0.6(0.13,3)$ \\
\hline pentamer & 8 & $\mathrm{CIC}$ & $-8.6 \pm 0.5$ & $0.5(0.08,3)$ \\
\hline pentamer & 9 & $\mathrm{CIC}$ & $-9.6 \pm 0.5$ & $0.09(0.02,0.4)$ \\
\hline trimer & 10 & $\mathrm{CIC}$ & $-3.7 \pm 0.2$ & $2000(1200,3000)$ \\
\hline trimer & 11 & $\mathrm{CIC}$ & $-4.8 \pm 0.8$ & $300(20,4000)$ \\
\hline trimer & 12 & $\mathrm{CIC}$ & $-6.6 \pm 0.3$ & $15(5,40)$ \\
\hline pentamer & 13 & DCI & $-4.4 \pm 0.4$ & $500(150,2000)$ \\
\hline pentamer & 14 & DCI & $-2.7 \pm 0.5$ & $10000(2000,50000)$ \\
\hline pentamer & 15 & DCI & $-4.4 \pm 0.5$ & $600(100,3000)$ \\
\hline pentamer & 16 & DCI & $-6.3 \pm 0.3$ & $20(9,60)$ \\
\hline pentamer & 17 & DCI & $-1.9 \pm 0.3$ & $30000(16000,90000)$ \\
\hline pentamer & 18 & DCI & $-4.0 \pm 0.2$ & $1100(500,2000)$ \\
\hline pentamer & 19 & DCI & $-10.4 \pm 0.5$ & $0.02(0.005,0.12)$ \\
\hline pentamer & 20 & DCI & $-3.7 \pm 0.3$ & $2000(700,6000)$ \\
\hline pentamer & 21 & DCI & $-4.7 \pm 0.4$ & $300(100,1400)$ \\
\hline trimer & 22 & DCI & $-3.2 \pm 0.2$ & $5000(2000,10000)$ \\
\hline trimer & 23 & DCI & $-1.8 \pm 0.3$ & $40000(15000,130000)$ \\
\hline trimer & 24 & DCI & $-6.8 \pm 0.4$ & $10(2,30)$ \\
\hline pentamer $(\mathrm{A} 25 \mathrm{~V})$ & 9 & $\mathrm{CIC}$ & $-8.7 \pm 0.6$ & $0.4(0.06,3)$ \\
\hline pentamer (A25V) & 19 & DCI & $-7.0 \pm 0.3$ & $7(2,20)$ \\
\hline pentamer (SARS-CoV2) & 9 & $\mathrm{CIC}$ & $-8.8 \pm 0.5$ & $0.4(0.07,2)$ \\
\hline pentamer (SARS-CoV2) & 19 & DCI & $-9.1 \pm 0.5$ & $0.2(0.04,1)$ \\
\hline
\end{tabular}

Table 1: Estimated binding free-energy $\Delta G$ for each multimer protein and ligand combination. CIC: ciclesonide, DCI: des-ciclesonide. Confidence intervals (CIs) are presented as pairs of (lower, higher) intervals. Mutimer proteins are MERS-CoV's nsp15 except the last two rows. 


\begin{tabular}{cccccccc}
\hline \multicolumn{3}{c}{ Ciclesonide pose 9 and a pentamer } & \multicolumn{5}{c}{ Des-ciclesonide pose 16 and a pentamer } \\
Chain & \# Res. & Res. name & $\%$ & Chain & \# Res. & Res. name & $\%$ \\
\hline A & 96 & Thr & 100 & A & 48 & Thr & 100 \\
A & 97 & Ala & 91 & A & 49 & Leu & 100 \\
A & 104 & Tyr & 100 & A & 50 & Pro & 100 \\
A & 105 & Thr & 93 & A & 96 & Thr & 100 \\
A & 107 & Ile & 100 & A & 97 & Ala & 100 \\
C & 34 & Lys & 89 & A & 104 & Tyr & 100 \\
D & 48 & Thr & 100 & A & 105 & Thr & 99 \\
D & 49 & Leu & 100 & A & 107 & Ile & 95 \\
D & 79 & Ile & 100 & C & 34 & Lys & 100 \\
D & 96 & Thr & 100 & C & 37 & Val & 100 \\
D & 97 & Ala & 99 & C & 39 & Asp & 100 \\
D & 104 & Tyr & 95 & D & 48 & Thr & 84 \\
D & 105 & Thr & 98 & D & 49 & Leu & 99 \\
D & 107 & Ile & 100 & D & 50 & Pro & 97 \\
D & 113 & Leu & 100 & D & 96 & Thr & 93 \\
& & & & D & 97 & Ala & 92 \\
& & & & D & 104 & Tyr & 100 \\
& & & & D & 105 & Thr & 100 \\
& & & & D & 106 & Asp & 81 \\
& & & & D & 107 & Ile & 100 \\
\hline
\end{tabular}

Table 2: Residues contacting with the ligand in more than $80 \%$ of the snapshots from gREST simulation. "\# Res." represents the residue number, and "Res. name" represents the residue name. 


\begin{tabular}{cccccc}
\hline \multicolumn{7}{c}{ Ciclesonide pose 9 and a pentamer } \\
Chain & Residue & Main/Side & Lig. atom & Water & $\%$ \\
\hline C & Lys34 & Side & O7 & no & 11 \\
D & Thr48 & Main & O3 & yes & 14 \\
D & Gly95 & Main & O6 & yes & 13 \\
D & Tyr104 & Main & O5 & yes & 26 \\
\hline \multicolumn{7}{c}{} \\
\hline \multicolumn{7}{c}{ Des-ciclesonide pose 16 and a pentamer } \\
Chain & Residue & Main/Side & Lig. atom & Water & $\%$ \\
\hline A & Tyr104 & Side & O4 & no & 13 \\
A & Thr48 & Main & O4 & yes & 20 \\
C & Gly36 & Main & O6 & yes & 10 \\
C & Val37 & Main & O6 & yes & 15 \\
D & Thr48 & Main & O4 & yes & 17 \\
D & Thr48 & Side & O3 & no & 52 \\
D & Thr96 & Main & O3 & yes & 15 \\
\hline \multicolumn{7}{c}{}
\end{tabular}

Table 3: Hydrogen bonds between the protein and (des-)ciclesonide after the gREST relaxation. Hydrogen bonds that were observed in more than $10 \%$ of the snapshots were listed. Chain represents the protein monomer symbols as presented in Fig. 2. Ligand atom names of oxygen atoms corresponds to the number on oxygen atoms in Fig. 1. The row of "Water" being "yes" indicates that protein-ligand has water-bridged hydrogen bonds, i.e., one water atom simultaneously make hydrogen bonds to both ligand and protein in a single snapshot. 